\title{
Host Rather than Graft Origin of Matrigel-Induced Adipose Tissue in the Murine Tissue-Engineering Chamber
}

\author{
FILIP STILLAERT, M.D., ${ }^{1,3}$ MICHAEL FINDLAY, M.B.B.S., B.Sc., FRACS, ${ }^{1,2}$ \\ JASON PALMER, B.Sc. (Hons), Dip. Ed., ${ }^{1}$ REJHAN IDRIZI, B.Sc. (Hons), ${ }^{1,2}$ \\ SHIRLEY CHEANG, M.B.B.S., ${ }^{1}$ AURORA MESSINA, Ph.D., ${ }^{1,2}$ \\ KEREN ABBERTON, M. Rep. Sci., Ph.D., ${ }^{1,2}$ WAYNE MORRISON, M.D.B.S., FRACS, ${ }^{1,2}$ \\ and ERIK W. THOMPSON, Ph.D.,
}

\begin{abstract}
We have recently shown that Matrigel-filled chambers containing fibroblast growth factor-2 (FGF2) and placed around an epigastric pedicle in the mouse were highly adipogenic. Contact of this construct with pre-existing tissue or a free adipose graft was required. To further investigate the mechanisms underpinning formation of new adipose tissue, we seeded these chambers with human adipose biopsies and human adipose-derived cell populations in severe combined immunodeficient mice and assessed the origin of the resultant adipose tissue after 6 weeks using species-specific probes. The tissues were negative for human-specific vimentin labeling, suggesting that the fat originates from the murine host rather than the human graft. This was supported by the strong presence of mouse-specific Cot-1 deoxyribonucleic acid labeling, and the absence of human Cot-1 labeling in the new fat. Even chambers seeded with FGF2/ Matrigel containing cultured human stromal-vascular fraction (SVF) labeled strongly only for human vimentin in cells that did not have a mature adipocyte phenotype; the newly formed fat tissue was negative for human vimentin. These findings indicate that grafts placed in the chamber have an inductive function for neo-adipogenesis, rather than supplying adipocyte-precursor cells to generate the new fat tissue, and preliminary observations implicate the SVF in producing inductive factors. This surprising finding opens the door for refinement of current adipose tissue-engineering approaches.
\end{abstract}

\section{INTRODUCTION}

\begin{abstract}
A DIPOSE-TISSUE ENGINEERING OFFERS improved treatment options in clinical practice by providing autologous tissue to restore tissue volume and surface contour. Successful tissue fabrication must include an appropriate environment for cell viability and function at the microscale level, as well as providing macroscale-level properties that allow sufficient transport of nutrients, provide adequate mechanical properties and tissue expansion, and facilitate coordination of multicellular processes. ${ }^{1}$ The new tissue construct should prosper
\end{abstract}

in equilibrium through biochemical, structural, and cellular cues and approximate the morphology and function of the native tissue equivalent.

Free-fat grafting rarely achieves sufficient tissue augmentation because of delayed neo-vascularization with subsequent cell necrosis, fibrosis, and graft volume shrinkage. It succeeds at a microscale level by providing the appropriate cell type in a suitable environment but fails at a macroscale level, because the majority of the transplanted cells become located more than $100 \mu \mathrm{m}$ away from blood vessels in the residual tissue, which is the diffusion limit for oxygen. The absence of

\footnotetext{
${ }^{1}$ Bernard Brien Institute for Microsurgery, Melbourne, Victoria, Australia.

${ }^{2}$ Department of Surgery, University of Melbourne, St Vincent's Hospital, Melbourne, Victoria, Australia.

${ }^{3}$ Department of Plastic and Reconstructive Surgery, University of Gent, University Hospital Gent, Gent, Belgium.
} 
more-suitable alternatives, such as tissue-engineering approaches, has led to its continued use despite variable resorption and lipid cyst formation.

A de novo engineered adipose tissue construct may be accomplished in vivo at the recipient site or cultured in vivo or in vitro. Hausberger found that new adipose tissue was deposited when grafts were transplanted in a newborn rat model. ${ }^{2}$ However, developing cells need an instructive matrix, and in natural tissues, the extracellular matrix (ECM) is known to provide structural stability, to orient and constrain cells during regeneration, and to act as a preserver of space for the new cells to develop. ${ }^{3}$ The ECM is actively remodeled during adipogenesis, with its geometrical architecture being an environmental cue for adipose tissue formation, ${ }^{3,4}$

Recent experimental models have used progenitor cells seeded onto ECMs or scaffolds in vitro, which are then implanted in vivo to generate bioartificial fat constructs. This strategy, called histioconduction, ${ }^{5-10}$ works on the premise that the 3-dimensional (3-D) scaffolds direct cell proliferation, differentiation, and organization into multicellular communities in such a way as a biohybrid, approximating the in vivo microarchitecture of functional subunits $(\sim 100 \mu \mathrm{m})$, the local microenvironment around cells $(\sim 10 \mu \mathrm{m})$, and the function of the wanted tissue, ${ }^{11}$ is produced. In practice, this approach is successful for the production of small volumes of tissue but has little clinical utility. The simple addition of cells to biocompatible scaffolds is inadequate for reproducing the biological and functional characteristics of the wanted tissue and fails to address the problem of vascularization of the biohybrid upon implantation, which significantly limits the size of the construct (and its clinical utility). This is especially true for tissues with a high metabolic demand, and tissue-engineering solutions might be more difficult to achieve for adipose tissue, which is strongly dependent on a functional vascular network.

An alternative for engineering adipose tissue constructs involves the induction of neovascularization of a cell-matrix scaffold, with concomitant recruitment of resident stem cells for the production of the target tissue (histioinduction). One matrix scaffold that has proven useful for experimental adipogenic histioinduction is Matrigel. ${ }^{12,13}$ Kawaguchi demonstrated that subcutaneously injected Matrigel supplemented with FGF2 (at a dose of $1 \mu \mathrm{g} / \mathrm{mL}$ Matrigel) induced de novo adipogenesis in mice. ${ }^{12}$ Angiogenic induction combined with the structural support provided by the Matrigel matrix appeared to create an ideal microenvironment for endogenous preadipocytes to migrate, proliferate, and differentiate. ${ }^{14}$ The stromal-vascular fraction (SVF) of adipose tissue is a wellknown source of multipotent adult stem cells and the presumed source of the recruited preadipocytes. ${ }^{15}$ Similarly, we found that Matrigel combined with FGF2 induced de novo angiogenesis and adipogenesis in a murine epigastric chamber model. ${ }^{16}$

Changes in the chamber configuration led to the observation that contact with existing fat tissue was essential for the adipogenic response to Matrigel to occur. ${ }^{17}$ The observation that chambers without access to any fat generated little or no adipose tissue, whereas the inclusion of an adipose graft within the sealed chamber with Matrigel gave reliable adipogenesis strengthened this hypothesis. We hypothesized that the addition of fat grafts to the sealed mouse chamber provided an adipogenic precursor cell source that combines with the Matrigel in the chamber to produce fat. To test this hypothesis, we added human adipose tissue (donor) to the chamber model using severe combined immunodeficient (SCID) mice (host) and used species-specific probes to determine whether the newly formed fat was of host or donor origin. We found clear evidence of host rather than graft origin, indicating that the Matrigel/FGF2-filled chamber, in conjunction with factors provided by the preexisting adipose tissue, recruits endogenous precursor cells that ultimately differentiate into new adipose tissue. This is an important refinement, given the popularity of adipose tissue grafting and the expectation that the adipose tissue grafts would contribute precursor cells.

\section{MATERIALS AND METHODS}

The St. Vincent's Hospital Animal Ethics Committee approved All animal experiments, which were conducted in accordance with the National Health and Medical Research Council (Australia) guidelines. Twelve-week-old SCID mice (minimum weight $18 \mathrm{~g}$, ARC, Perth, Australia) were used in this study and maintained in Techniplast individually ventilated cages (Milan, Italy) with standard chow and water ad libitum.

\section{Materials}

Cylindrical chambers were fashioned from 5-mm lengths of laboratory tubing (Dow-Corning Corp., Midland, MI), with an internal diameter of $3.35 \mathrm{~mm}$ and a volume of $44 \mu \mathrm{L}$, as previously described. ${ }^{16,17}$ Dulbecco's modified Eagles medium (DMEM), penicillin G, and streptomycin sulfate were purchased from Gibco/Invitrogen Life Technologies Corporation (Glen Waverley, Australia). Phosphate buffered saline (PBS) solution was purchased from Oxoid Ltd. (Hampshire, England). Trypsin and fetal calf serum (FCS) were purchased from CSL Ltd. (Melbourne, Australia). Matrigel (complete Matrigel unless specified as growth factor reduced (GFR)) was obtained from Becton \& Dickinson (North Ryde, Australia).

\section{Fat graft preparation}

Human adipose biopsies were obtained with informed preoperative patient consent through the Plastic and Reconstructive Surgery Unit at St Vincent's Hospital, Melbourne, with approval from the St Vincent's Hospital Human Research Ethics Committee. Specimens were obtained from the abdominal region during reconstructive surgery and trans- 
ported on ice in DMEM. Excess fibrous tissue was dissected under aseptic conditions, and specimens were randomly minced into small cubes of 1 to $3 \mathrm{~mm}^{3}$ before being washed twice in fresh DMEM. The selected cubes were then sealed separately in preweighed Eppendorf tubes containing DMEM and kept on ice until implantation. The weight of each specimen was recorded before implantation.

\section{Matrigel-enriched cell cultures}

Forty-eight-well tissue culture plates (Becton \& Dickinson) were coated with GFR Matrigel supplemented with $1 \mu \mathrm{g} / \mathrm{mL}$ FGF2 (Sigma Aldrich, Sydney, Australia) and $80 \mathrm{U} / \mathrm{mL}$ of unfractionated sodium heparin (APP Inc, Schaumburg, IL) and allowed to set at $37^{\circ} \mathrm{C}$ for 2 to $3 \mathrm{~min}$. Tissue cubes 2 to $3 \mathrm{~mm}^{3}$ in size were dissected from crude fat biopsies, assessed for degree of SVF content, and placed onto the Matrigel-coated wells before gelation, and the whole plate was incubated for 2 to 3 minutes at $37^{\circ} \mathrm{C}$. The wells were then filled with $0.5 \mathrm{~mL}$ of DMEM supplemented with $10 \%$ FCS, $2.92 \mu \mathrm{g} / \mathrm{mL}$ L-glutamine, and $100 \mu / \mathrm{mL}$ penicillin $\mathrm{G} /$ streptomycin sulfate and the plates incubated at $37^{\circ} \mathrm{C}$ in a humidified, $5 \%$ carbon dioxide $\left(\mathrm{CO}_{2}\right)$ atmosphere for 4 to 8 weeks. Medium was changed every 3 to 4 days. These are refereed to as MA cultures.

\section{SVF culture}

Large fibrous tissues were carefully dissected from the fat biopsy. The remaining tissue was digested with $0.2 \%$ collagenase (Type I, Worthington Pty Ltd., Lakewood, NJ) in PBS with shaking at $37^{\circ} \mathrm{C}$. Large tissue chunks were removed using a $100-\mu \mathrm{m}$ cell strainer. Samples were pelleted in a standard cell culture centrifuge at room temperature and washed 3 times with PBS. Cell pellets were resuspended in DMEM with $10 \%$ FCS and antibiotics as described above. After counting, cells were plated into tissue culture flasks at approximately $2.1 \times 10^{6}$ cells per $75-\mathrm{cm}^{2}$ flask. Cultures were then incubated at $37^{\circ} \mathrm{C} 5 \% \mathrm{CO}_{2}$. Non-adherent red blood cells were removed with 3 PBS washes on the following day. The resultant SVF was cultivated and used within 3 passages.

\section{The flow-through loop pedicle murine chamber model}

The in vivo model used was essentially as previously described. ${ }^{16,17}$ Briefly, the mice were anesthetized with an intraperitoneal injection of chloral hydrate $(0.4 \mathrm{mg} / \mathrm{g}$ body weight), and the groin and upper legs were depilated. Silicon chambers were inserted bilaterally in both groins, with the superficial inferior epigastric vessels being dissected free from the surrounding tissue, and then encased by the chamber, which was opened along one side to allow incorporation of the vessels. The chamber was sealed at the femoral end and along the lateral split with melted Ethicon bone wax (Johnson \& Johnson International, St Stevens-Woluwe, Belgium). The entire chamber was then anchored to the underlying muscle with a 10/0 nylon suture (Davis \& Geck,
St Louis, MO) to prevent dislodgement during postoperative mobilization of the animals. The chamber was then filled with Matrigel, the fat biopsy or cells were placed in the Matrigel, and the epididymal end was sealed with melted bone wax, avoiding any excessive heating or obstruction of the vascular pedicle. Skin incisions were closed with surgical clips.

\section{Harvest of tissue}

There were 5 experimental groups (A-E), all containing Matrigel and all harvested at 6 weeks postimplantation, except group B (9-week harvest). Groups A ( $n=13$ chambers) and $\mathrm{B}(n=8$ chambers $)$ contained human adipose tissue autografts, Group C ( $n=15$ chambers) had established MA cultures (with or without original fat biopsies) added to the chambers instead of fat biopsies, Group D ( $n=6$ chambers) had SVF culture rather than biopsy added to the chamber, and Group E ( $n=8$ chambers) were Matrigel-only controls. Chambers were removed under aseptic conditions at 6 or 9 weeks postimplantation under the same anesthetic conditions used for chamber insertion, and the mice were then killed using a lethal intraperitoneal dose of $0.3 \mathrm{~mL}$ of Lethobarb (Pentobarbitone, Virbac, NSW, Australia). During chamber removal, the patency of the vascular pedicle was assessed by looking for evidence of bleeding from the vessel ends when the pedicles were cut where they exited the chamber. The chamber could then be removed from its surrounding capsule and its contents assessed. Chamber tissues were all weighed and measured using standard volumedisplacement techniques. Specimens were fixed overnight in $10 \%$ buffered formalin, dehydrated, and embedded in paraffin wax in the standard manner.

\section{Morphological analysis}

Samples were cut into 5- $\mu \mathrm{m}$ sections, mounted onto slides, and stained with Masson's Trichrome to assist with identification of adipose tissue (mature adipocytes), connective tissue, angiogenesis (endothelial cells/new blood vessels), biopsy (original cells from the fat biopsy), vascular pedicle, and remnants of Matrigel in the sections using point counting. Every 20th section throughout each specimen was point counted, as previously described, ${ }^{16,17}$ using the Computer Assisted Stereological ToolBox system (Olympus Denmark $\mathrm{A} / \mathrm{S}$, Albertsland, Denmark). The adipose tissue percentage of each specimen was determined dividing the number of points containing adipose tissue by the total number of points containing tissue, and multiplying by 100 .

\section{Vimentin immunolocalization}

Immunolabeling of human vimentin in these sections was performed using the Dako ARK (Animal Research Kit, Dako, Carpinteria, CA). Sections were dewaxed, rehydrated, incubated in Dako target retrieval buffer $(\mathrm{pH} 6.0)$ at $95^{\circ} \mathrm{C}$ for 
$20 \mathrm{~min}$, and then left to cool at room temperature for the same time. All subsequent steps took place at room temperature, and washes between steps were performed with PBS. Endogenous peroxidase activity was quenched with $3 \%$ hydrogen peroxide for $5 \mathrm{~min}$, the primary antibody (Dako mouse antivimentin, clone V9) was biotinylated according to kit instructions, and $100 \mu \mathrm{L}$ was applied to sections at 1:600 for $30 \mathrm{~min}$. Sections were then incubated with streptavidinperoxidase for $30 \mathrm{~min}$, followed by 3,3-diaminobenzidine chromogen solution (Dako) for $5 \mathrm{~min}$. They were then counterstained with Mayer's hematoxylin, dehydrated, and mounted in Depex (BDH Chemicals, Kilsyth, Australia). Confirmation of vimentin reactivity in mouse cells was performed using a second vimentin antibody (Abcam rabbit anti-vimentin, ab7783). Dewaxing, hydration, antigen retrieval, and peroxidase quenching were performed as described above. Non-specific binding was blocked using $10 \%$ normal swine serum (Dako) and the primary antibody diluted 1:300 in buffer with $1 \%$ bovine serum albumin then applied. This was followed with swine anti-rabbit biotin (Dako), 1:400 for $30 \mathrm{~min}$; horseradish peroxidase-streptavidin (Dako), 1:400 for $30 \mathrm{~min}$; and 3,3-diaminobenzidine and counterstaining, etc., as above.

\section{Fluorescent in situ hybridization for Cot-1 deoxyribonucleic acid}

The mouse and human Cot-1 deoxyribonucleic acid (DNA, Molecular Probes, Invitrogen, Glen Waverley, Australia) were labeled with digoxigenin (DIG) using a DIGnick translation mix (Roche, Germany). Briefly, the standard labeling reaction involved adding $1 \mu \mathrm{g}$ of template DNA to $16 \mu \mathrm{L}$ of sterile redistilled water and then adding $4 \mu \mathrm{L}$ of DIG-nick translation mix and incubating for $90 \mathrm{~min}$ at $15^{\circ} \mathrm{C}$. After the incubation, the reaction was placed on ice, and a small sample was run on an agarose mini-gel along with DNA molecular weight markers to ensure that the probe was between 200 and 500 nucleotides in length. When the correct probe length was achieved, the reaction was stopped by adding $1 \mu \mathrm{L} 0.5 \mathrm{M}$ ethylenediaminetetraacetic acid ( $\mathrm{pH}$ 8.0) and heating to $65^{\circ} \mathrm{C}$ for $10 \mathrm{~min}$. Sections were deparaffinized in xylene for $10 \mathrm{~min}$, dehydrated, and air-dried. They were then incubated in $1 \mathrm{M}$ sodium thiocyanate for $10 \mathrm{~min}$ at $80^{\circ} \mathrm{C}$, washed in PBS, and digested with proteinase $\mathrm{K}(20 \mu \mathrm{m} / \mathrm{mL})$ for $10 \mathrm{~min}$ at $37^{\circ} \mathrm{C}$. After quenching in $0.2 \%$ glycine solution, they were washed in PBS, postfixed in $4 \%$ paraformaldehyde, dehydrated in graded alcohols, and air-dried. DIG-labeled mouse or human Cot-1 DNA was then applied to sections and sealed under glass coverslips. Simultaneous denaturation was performed on a preheated hot plate set to $60^{\circ} \mathrm{C}$, followed by incubation overnight at $37^{\circ} \mathrm{C}$ in a humidified chamber. Slides were washed sequentially in $50 \%$ formamide $/ 2 \times$ standard saline citrate $(\mathrm{SSC})$ for $3 \times 5 \mathrm{~min}$ each, followed by $2 \times \mathrm{SSC}$ alone $(3 \times 5 \mathrm{~min}$ each $)$ and then $4 \times \mathrm{SSC} /$ $0.05 \%$ Tween 20 for $10 \mathrm{~min}$ at $37^{\circ} \mathrm{C}$. Slides were then washed in PBS, incubated in blocking solution for $30 \mathrm{~min}$, and incu- bated sequentially in the following antibody solutions (all antibodies at a concentration of $1: 25$ ) for $60 \mathrm{~min}$ each at $37^{\circ} \mathrm{C}$ with 5 -min washes in $0.2 \%$ Tween 20 in $1 \times$ PBS between each incubation: anti-DIG monoclonal antibody, antimouseimmunoglobulin-DIG, $\mathrm{F}\left(\mathrm{ab}^{\prime}\right)$ 2-fragment, and anti-DIGfluorescein. Sections were washed with PBS and then distilled water, counterstained using 4,6-diaminidino-2-phenylindone (DAPI) at $40 \mathrm{ng} / \mathrm{mL}$, and then mounted in Vectashield antifade solution (Vector Laboratories, Burlingame, CA). The slides were examined using a fluorescent microscope (Zeiss, North Ryde, NSW, Australia). Positive signals for the Cot-1 DNA were identified as green dots within blue DAPI counterstained nuclei.

\section{RESULTS}

\section{Generation of adipose tissue}

Matrigel/FGF2-only control chambers. The overall adipogenic response in the control chambers containing FGF2supplemented GFR Matrigel but no inductive graft was poor, consistent with previous findings. ${ }^{17}$ After 6 weeks of incubation, isolated adipocytes and fibroblast-like cells were seen scattered within the Matrigel, but few clusters of adipocytes were seen (Fig. 1A). The percentage of mature fat cells calculated by point counting in the control chambers averaged $17 \%$. These adipocytes may have originated from the remnants of the perivascular fat pad, having migrated and differentiated within the Matrigel environment with concomitant sprouting of neo-capillaries from the murine vascular pedicle.

Matrigel/FGF2 chambers with human fat biopsies. Group A (6 weeks) and B (9 weeks) chambers, which received a human fat biopsy in addition to the FGF2-supplemented Matrigel, both generated vascularized 3-D adipose tissue constructs (Fig. 1B,C). The overall adipogenic response in the biopsy-seeded chambers was better than that of the control chambers, with significant variation in adipogenic induction between the engineered fat constructs $(p=.05)$, as assessed using one-way analysis of variance with Dunnett's post hoc analysis. The average adipocyte counts were $30 \%$ (6 weeks) (Fig. 1D) and 28\% (9 weeks) (Fig. 1E).

In Group A, of the 13 total chambers established, 4 were excluded because of infection $(n=2)$ and pedicle thrombosis $(n=2)$, leaving 9 chambers for inclusion in the study. Sprouting neo-capillaries seemed to direct specifically toward the biopsy, particularly when the latter contained a higher proportion of SVF to adipose tissue (Fig. 2A). The degree of adipogenesis varied strongly throughout the chambers, with adipogenic differentiation concentrated around (neo-) capillaries (Fig. 2B). Two trends were apparent, although neither was formally assessed. Human fat biopsies containing a higher proportion SVF appeared to induce better adipogenic responses than biopsies containing minimal SVF, the latter inducing poor to moderate adipogenic responses, 


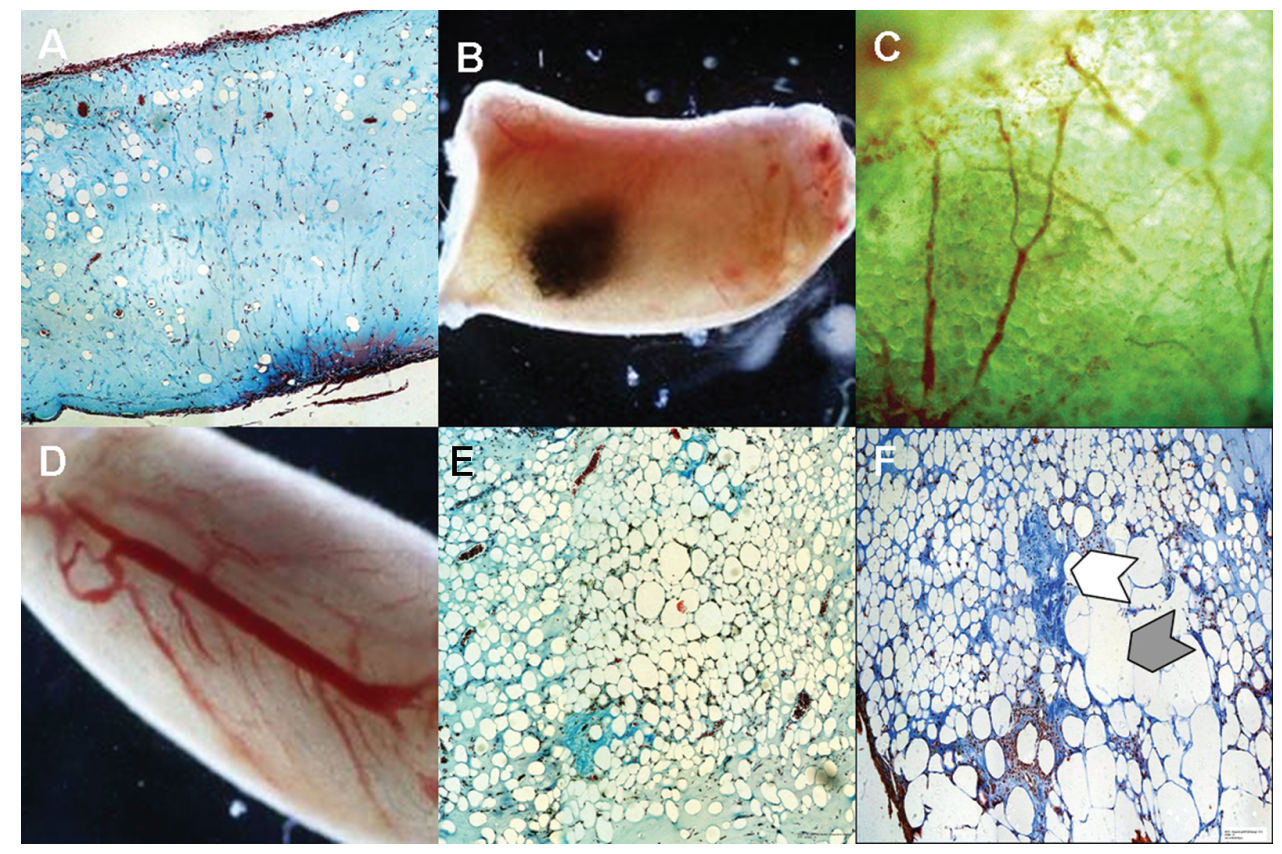

FIG. 1. (A) Masson's Trichrome-stained longitudinal section from a Matrigel-only control chamber. Only few scattered isolated mature adipocytes can be found in the predominantly Matrigel-filled chamber. (B) Macroscopic view of a fat construct harvested at 6 weeks from a Matrigel/fibroblast growth factor (FGF)2-supplemented chamber. The original fat biopsy had been labeled with India ink before implantation in the chamber. (C) Inferior view of the main host vascular pedicle with angiogenesis sprouting from the epigastric pedicle. (D) Detailed view of the surface of one of the generated fat constructs at 6 weeks. Neo-capillaries are observed surrounding mature adipocytes that is translated in the typical cobblestone appearance of fat tissue. Group A (6 weeks) (E) and B ( 9 weeks) (F) chambers, which received a human fat biopsy in addition to the FGF2-supplemented Matrigel, both generated adipose tissue. The original fat graft adipocytes degenerated into lipid cysts (gray arrow) and the stromal-vascular fraction is observed within the original fat xenograft (white arrow). Neocapillaries are seen penetrating the Matrigel environment (black arrow). Color images available online at www.liebertpub.com/ten.

and there did not appear to be a direct correlation between the size of the original human fat biopsies and the adipogenic response.

In Group B, of the 8 chambers set up, one was excluded because one of the wax plugs sealing the chamber had become dislodged, and murine fat tissue clearly invaded the chamber contents. The 7 remaining chambers were viable, and all generated adipose tissue. Levels of adipogenesis again varied between the chambers.

Chambers containing MA cultures (Group C). Angiogenesis was well developed throughout chambers containing MA cultures. Numerous small vessels were clearly seen sprouting from the pedicle along the periphery of the chamber,

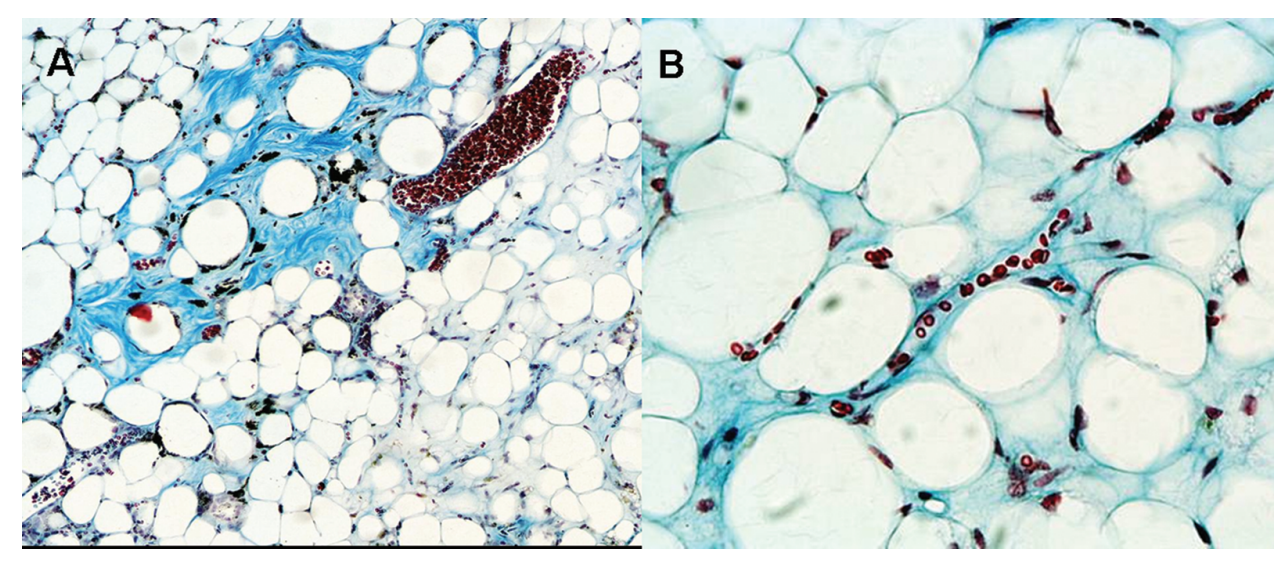

FIG. 2. (A) Neo-capillaries directed specifically towards the stromal-vascular fraction of the fat biopsy. (B) A close association between developing adipocytes and sprouting young capillaries was observed in chambers generating adipose tissue. Color images available online at www.liebertpub.com/ten. 
and new vessels and capillaries could be observed in most parts of the chamber tissue. Approximately 25\% of the chambers were filled with small uni- and multilocular adipocytes. The rest of the chamber contents included the original pedicle, residual Matrigel-supporting elongated cells, the original crude biopsies from the cultures, and a fibrous tissue capsule.

Chambers containing SVF cultures (Group D). Tiny vessels sprouting from the embedded pedicle were seen macroscopically in chambers seeded with SVF cultures. Histologically, numerous mature adipocytes were scattered around the chambers, contributing $30 \%$ to $40 \%$ to the chamber content. Multilocular adipocytes, elongated cells, bluestaining collagen fibers, and multilayered capsule formation were observed, as well as pedicle tissues and residual Matrigel. However, chambers seeded with SVF cultures appeared less vascularized than the MA-seeded chambers, suggesting that the less-purified cultures of MA contained higher levels of angiogenic factors.

\section{Identification of the origin of the generated fat tissue: vimentin immunolocalization}

Biopsy chambers. Staining of the newly generated adipocytes in the engineered constructs for the intermediate filament protein vimentin using a human-specific antibody was negative. However, vimentin expression was confirmed in these cells with a mouse-specific antibody, and there was prominent human vimentin labeling of the interstitial fibroblast-like cells in the SVF of the xenografts (Fig. 3 A, B).

$M A / S V F$ chambers. Vimentin labeling was positive in the human fibroblast-like cells of SVF culture chambers in vitro when embedded in paraffin and sectioned, and some labeling of mature adipocytes was also observed in the crude tissue biopsies (Fig. 4). In contrast, there was no labeling of new adipocytes within MA or SVF chambers, although some fibroblast-like cells in the chambers labeled positive for vimentin. Resting and migrating macrophages also labeled positive for vimentin in some chambers, probably as a result of digesting necrotic cells.

Cot-1 DNA labeling. Most of the generated adipose tissue was strongly positive for the mouse-specific Cot-1 DNA using the fluorescent in situ hybridization technique, whereas there was a distinct absence of any human Cot-1 DNA labeling, confirming the murine origin of the new adipocytes (Fig. 5).

\section{DISCUSSION}

Cell transplantation techniques focus on direct injection of cells or on placing them on biodegradable scaffolds before implantation in vivo. In the field of adipose-tissue engineering, the purpose is to generate 3-D cultures on scaffolds before implantation, but this technique has practical disadvantages because of diffusion limitations for cell survival. Transplantation of cells must be augmented with methods to ensure cell survival, such as the induction of an angiogenic framework. ${ }^{18}$ In vivo cell recruitment (histioinduction) is one of the techniques currently being explored at our institute to engineer adipose tissue. This approach seems to be rational, because it could induce concomitantly adipo- and angiogenesis. The experimental in vivo model we are currently using has the advantage that those 2 histiogenic processes can be induced simultaneously. The Matrigel matrix supports the interplay, or cross-talk, between developing adipocytes and endothelial cells, which are not in direct apposition to each other.

The studies described here confirm and extend our previous reports of the adipogenic potential of Matrigel in our chambers. ${ }^{16,17}$ Sealed, Matrigel-filled chambers containing excised syngeneic fat, cultured SVF cells, or 3-D explants of cultured adipose biopsy produced 3-D vascularized tissue constructs with a higher percentage of fat than the control chambers with Matrigel alone. This is consistent with previous findings in $\mathrm{C} 57 \mathrm{bL} / 6$ mice, in which access to preexisting tissue was essential for de novo adipogenesis in

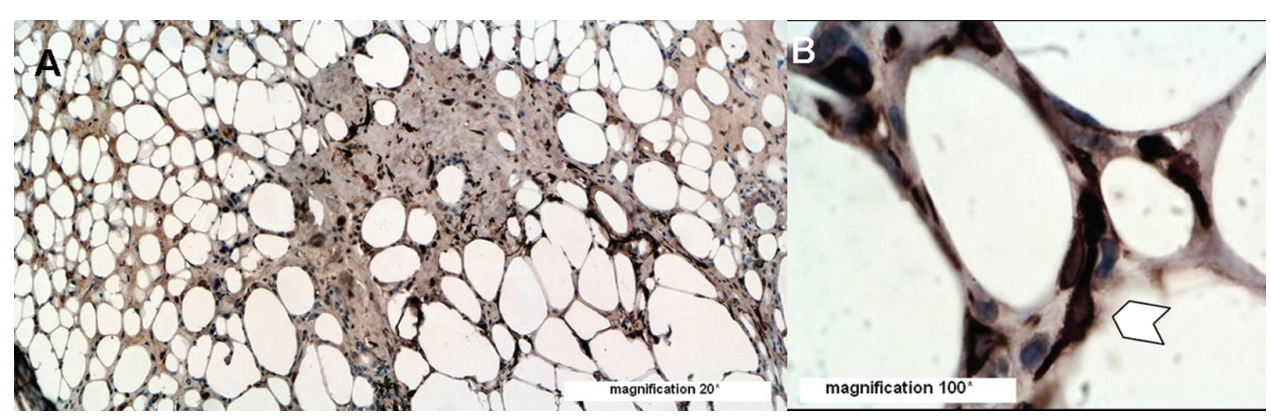

FIG. 3. (A) Human-specific vimentin labeling shows positive fibroblast-like cells within the stromal-vascular compartment of the original fat xenograft, suggesting survival of those cells, presumable preadipocytes (magnification $\times 20$ ). (B) Detailed view of the cells in the xenograft. Positive fibroblast-like cells are seen between degenerated adipocytes. Color images available online at www .liebertpub.com/ten. 


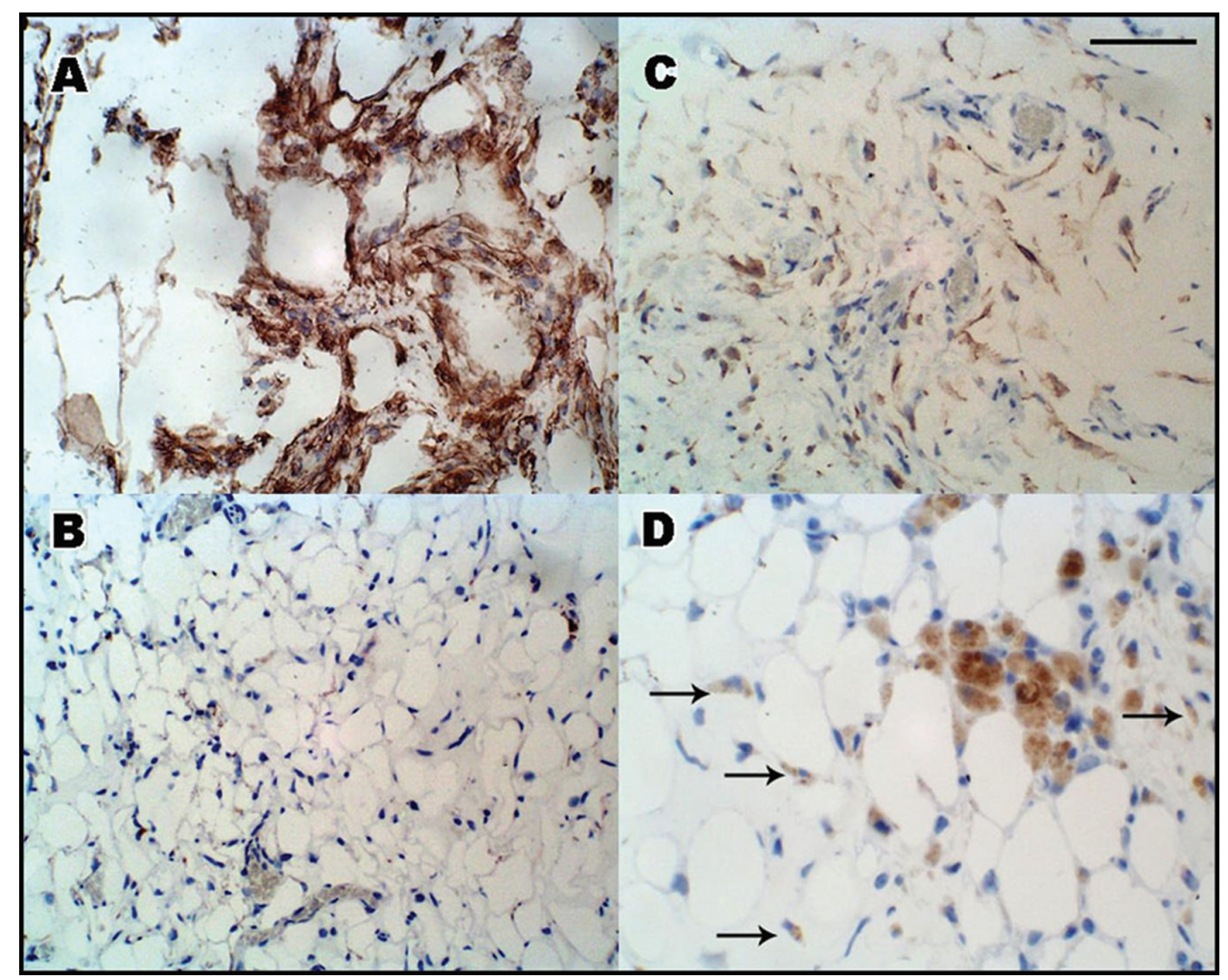

FIG. 4. Vimentin labeling of human stromal-vascular fraction (SVF) cultures in vitro and in vivo. (A) In vitro cells from SVF cultures labeled strongly for vimentin. (B) Adipocytes arising within in vivo chambers implanted with these cultures were almost all negative. (C) Fibroblastic cells in chambers implanted with SVF cultures, labeled for vimentin. (D) Migrating macrophages (arrows), which resembled positive-staining fibroblastic cells, around a group of resting macrophages. Color images available online at www.liebertpub.com/ten.

the Matrigel/FGF2-filled chambers. ${ }^{16,17}$ Also, in studies in which Matrigel induced fat in the subcutaneous space, access to fat was constitutive, and the adipogenic precursors were host derived. ${ }^{12,13,19}$ Labeling with human- and mouse- specific probes for Cot-1 DNA and vimentin immunolabeling confirmed the host derivation of the new adipose tissue, indicating that the requirement for contact with adipose tissue relates to inductive factors rather than the provision of

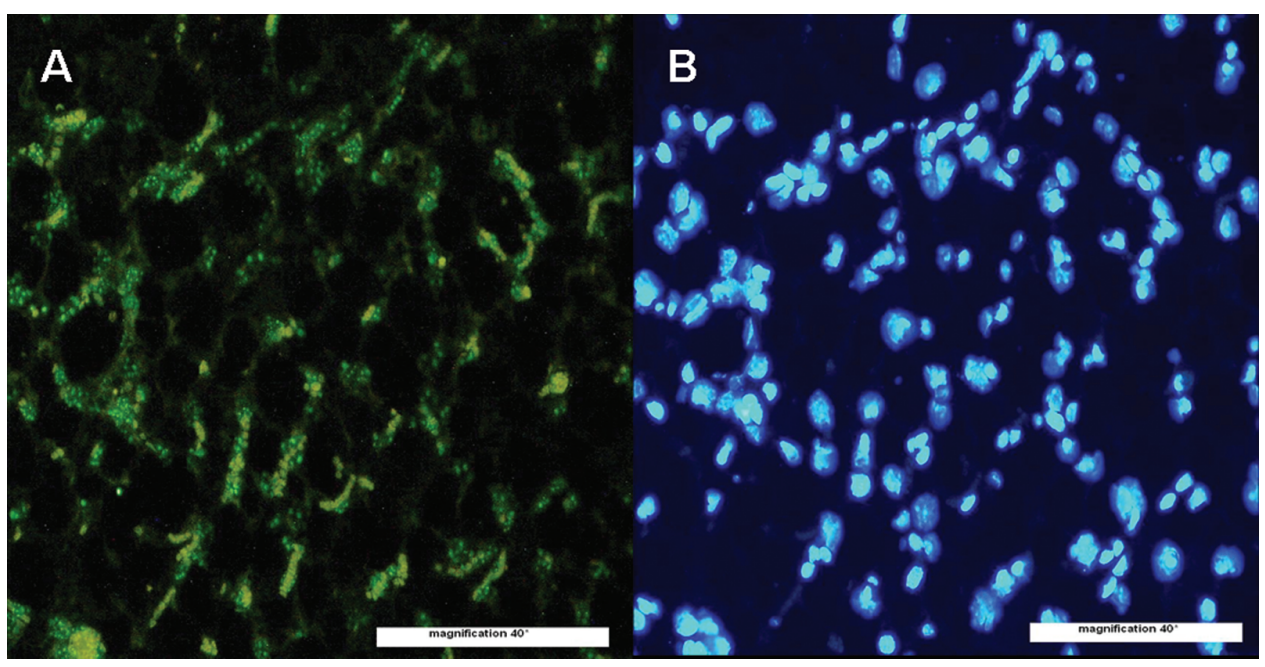

FIG. 5. Most of the generated adipose tissue was strongly positive for mouse specific Cot-1 deoxyribonucleic acid (DNA) (A) using the fluorescent in situ hybridization technique, whereas there was a distinct absence of any human Cot-1 DNA labeling, confirming the murine origin of the new adipocytes. (B) 4,6-diaminidino-2-phenylindone counterstain (scale bar $=50 \mu \mathrm{m}$ ). Color images available online at www.liebertpub.com/ten. 
precursor cells. Indeed, the likelihood that the cells that form the new adipose tissue represent mesenchymal stem cells (MSCs) is high, because these cells are pluripotent and can generate adipose tissue with appropriate signals. The combination of Matrigel, FGF2, and an inductive graft cause the recruitment or differentiation of such host-derived precursor cells.

Bone marrow is a rich source of circulating precursor cells, including endothelial precursor cells and bone marrow MSCss. ${ }^{20}$ Although bone marrow MSCs are a small constituent of the bone marrow, chemokines released in response to cells and tissue hypoxia can recruit them..$^{20}$ Another potential source of adipogenic precursor cells may be vascular pericytes or host preadipocytes that may be present surrounding the pedicle despite careful surgical stripping. This may explain the small amount of adipogenesis seen in the Matrigelonly group. The smaller amounts of adipogenesis seen in these chambers than in those containing grafts confirmed the adipo-inductive effect of the graft. Recent studies, ${ }^{15,21}$ as well as our own work (Ramakrishnan, Morrison et al., unpublished observation), show that adult adipose tissue also houses MSCs with similar pluripotential characteristics. We found that human MSCs purified from human lipoaspirate or bone marrow induced fat in SCID mouse chambers and that this new fat was also of recipient origin, although the vasculature was of mixed recipient and human origin.

Thus, it is surprising that the biopsy-derived MSCs do not contribute to the new adipose tissue, especially given the apparent association between SVF and degree of adipogenesis. One important caveat is a possible species mismatch between an essential cytokine and receptor, such as that known to exist for macrophage colony-stimulating factor receptor (c-fms) and colony stimulating factor-1, which causes mouse colony stimulating factor- 1 not to recognize the human form of the c-fms receptor. Another caveat would be an important role for the immune effector cells, which are lacking in SCID mice, especially because these have been implicated in adipogenesis. However, we recently performed similar studies transplanting male biopsies into female hosts, and vice-versa, in intact syngeneic mice, and preliminary analysis reveals a similar scenario of hostderived adipose tissue in response to an inductive graft (Thomas et al., manuscript in preparation).

In considering the nature of the inductive factor(s), it is noteworthy that we observed better adipose outcomes when free-fat flaps containing a higher proportion of adipose tissue were used. Biopsies containing more SVF also demonstrated better cell survival in that compartment of the graft, consistent with positive labeling for vimentin seen in these regions of the biopsies. A common factor in the three types of inductive sources used in all of these experiments (graft, SVF culture, and MA culture) is the presence of a SVF component. Cultured exogenous SVF/preadipocytes have been used to induce fat subcutaneously, in conjunction with other scaffolds. ${ }^{13}$ Kimura et al. have reported that, when collagen sponge seeded with human SVF was implanted subcutaneously into nude mice, they achieved human adipose tissue, as indicated by vimentin immunolabeling. These data contrast with our findings in the chamber environment, which may be different because of the subcutaneous environment or the type of scaffold.

The in vitro cultures of SVF or MA in the chambers induced different tissue morphologies than in chambers seeded with a fat graft. These changes, which appear to include greater adipogenesis, may relate to the presence of more SVF cells than in whole adipose biopsies. In vitro culturing of SVF-residing cells with their intrinsic heterogeneity could also have upgraded them into a further stage of more-mature cell development. These chambers also appeared to show better qualitative vascularization than the chambers containing fat biopsies. It may be that the additional cell fraction present encouraged a faster rate of angiogenesis, leading to a greater chance of cell survival. Cellular structures resembling tubules were observed in the chambers seeded with MA cultures, suggesting that ECM components may have been modified in culture or that the cells may secrete additional ECM components and that these ECM components were able to act as inductive factors to further enhance adipogenesis in vivo, similar to previous in vitro studies. ${ }^{22}$ Prolonged in vitro culture of MA tissue and cells could alter cell behavior, also affecting adipogenesis when seeded into chambers. The presence of growth or differentiation factors secreted by the cultured cells may be more important than the presence of the cells themselves. Factors such as vitronectin ${ }^{23}$ and FGF2 that have roles in regulating adipogenesis and angiogenesis could supplement host-derived adipogenesis response to Matrigel. Further studies will be undertaken to assess the potential benefits of culturing autologous SVF for implantation.

Mature adipocytes introduced in all adipose biopsies degenerated into cyst-like lipid deposits. Differences between implanted biopsies, such as in the ratio of stromal tissue to mature adipocytes appeared to affect the proportion of adipose tissue and other components in individual chambers. Adipocytes and preadipocytes represent less than $50 \%$ of the total cells in adipose tissue; the remaining cells include stromal cells, blood cells, endothelial cells, and pericytes. Some investigators have indicated that only $0.02 \%$ of the SVF are preadipocytes capable of adipogenic differentiation, ${ }^{24}$ with the rest of the population consisting of endothelial cells and fibroblasts.

A strong relationship has been observed between angiogenesis and adipogenesis in the chamber model. ${ }^{17}$ The fat graft appeared to attract capillaries sprouting from the main pedicle in the chambers, suggesting that biopsy-released factors are initiating the neovascularization process, probably via hypoxia-induced factors. ${ }^{20}$ Hypoxia appears to be an important regulator of adipocytes and endothelial cells. Vascular endothelial growth factor, an important proangiogenic factor, is released from endothelial cells during 
hypoxia and plays a role in signaling between endothelial cells and preadipocytes. ${ }^{25}$ Such coordination between endothelial cells and preadipocytes may maintain the adipose precursor cell population in a nondifferentiated, ischemiatolerant state until sufficient vascularization has occurred. The implantation of a whole tissue graft containing preadipocytes and endothelial cells in the SVF has the advantage of preserving the original cell structures, allowing for the subsequent interplay between those different cell populations during the initial hypoxic period in the Matrigelfilled tissue-engineering chamber. The SVF region in the xenograft could function as a source of vasoactive signals due to intercellular signaling processes between preadipocytes, adipocytes, and endothelial cells. The surrounding Matrigel environment may further enhance the response to these signals due to selective binding of growth factors and its action as a permissive substratum for recruited cells.

\section{CONCLUSIONS}

Our studies have considerable bearing on the growing number of approaches to adipose engineering, such as use of inductive fat with auto-grafted "preadipocytes" or preadipocyte-conferring components such as processed lipoaspirate. ${ }^{6,15}$ These have traditionally been thought to provide a source of new preadipocytes, which differentiate into adipocytes, although our studies suggest otherwise. It may also shed further light on the question of whether it is graft survival, de novo adipogenesis, or a combination of both that occurs during autologous fat transplantation, with significance for our understanding of why such variable results are obtained with this widely used technique. In conclusion, we have produced 3-D vascularized adipose constructs using crude adipose biopsies or stromal-vascular cell cultures. Species-specific labeling indicates that the new tissue is of host origin, rather than from the donor explants. The host origin of the newly derived adipogenesis suggests that the explants are acting in an inductive capacity, rather than as a source of exogenous precursor cells for the new tissue. These findings will be important in determining the optimum conditions for the development of de novo fat tissues for tissue engineering.

\section{ACKNOWLEDGMENTS}

We gratefully acknowledge surgical assistance from Sue McKay, Liliana Pepe, Anna Deftereos, and Amanda Rixon from the Experimental Medical Surgical Unit, St Vincent's Hospital, Melbourne. Thanks also to Ms Effie Keramidaris for assistance with the in vitro tissue culture experiments and John Rophael for his contribution to animal surgery. Grant support from the NH \& MRC, Australia (\#299872) is gratefully acknowledged.

\section{REFERENCES}

1. Tsang V. L, Bhatia S.N. Three-dimensional tissue fabrication. Adv Drug Deliv Rev 56, 1635, 2004.

2. Hausberger FX. Quantitative studies on the development of autotransplants of immature adipose tissue of rats. Anat Rec 122, 507, 1955.

3. Kleinman HK, Philp D, Hoffman MP. Role of the extracellular matrix in morphogenesis. Curr Opin Biotechnol 14, 526, 2003.

4. Nakajima I, Yamaguchi T, Ozutsumi K, Aso H. Adipose tissue extracellular matrix: newly organized by adipocytes during differentiation. Differentiation 63, 193, 1998.

5. Kral JG, Crandall DL. Development of a human adipocyte synthetic polymer scaffold. Plast Reconstr Surg 104, 1732, 1999.

6. Patrick CW Jr, Chauvin PB, Hobley J, Reece GP. Preadipocyte seeded PLGA scaffolds for adipose tissue engineering. Tissue Eng 5, 139, 1999.

7. Huss FR. Kratz G. Mammary epithelial cell and adipocyte co-culture in a 3-D matrix: the first step towards tissueengineered human breast tissue. Cells Tissues Organs 169, 361, 2001.

8. von Heimburg D, Zachariah S, Heschel I, et al. Human preadipocytes seeded on freeze-dried collagen scaffolds investigated in vitro and in vivo. Biomaterials 22, 429, 2001.

9. Patrick CW Jr, Zheng B, Johnston C, Reece GP. Long-term implantation of preadipocyte-seeded PLGA scaffolds. Tissue Eng 8, 283, 2002.

10. Kang X, Xie Y, Kniss DL. Adipose tissue model using threedimensional cultivation of preadipocytes seeded onto fibrous polymer scaffolds. Tissue Eng 11, 458, 2005.

11. Langer R. Vacanti. J.P. Tissue engineering. Science 260, 920, 1993.

12. Kawaguchi N, Toriyama K, Nicodemou-Lena E, Inou K, Torii S, Kitagawa Y. De novo adipogenesis in mice at the site of injection of basement membrane and basic fibroblast growth factor. PNAS 95, 1062, 1998.

13. Kimura Y, Ozeki M, Inamoto T, Tabata Y. Time course of de novo adipogenesis in matrigel by gelatin microspheres incorporating basic fibroblast growth factor. Tissue Eng 8, 603, 2002.

14. Toriyama K, Kawaguchi N, Kitoh J, et al. Endogenous adipocyte precursor cells for regenerative soft-tissue engineering. Tissue Eng 8, 157, 2002.

15. Zuk PA, Zhu M, Ashjian P, et al. Human adipose tissue is a source of multipotent stem cells. Mol Biol Cell 13, 4279, 2002.

16. Cronin KJ, Messina A, Knight KR, et al. New murine model of spontaneous autologous tissue engineering, combining an arteriovenous pedicle with matrix materials. Plast Reconstr Surg 113, 260, 2004.

17. Kelly JL, Findlay MW, Knight KR, et al. Contact with existing adipose tissue is inductive for adipogenesis in matrigel. Tissue Eng 2, 2041, 2006.

18. Miller MJ, Patrick CW Jr. Tissue engineering. Clin Plast Surg 30, 91, 2003.

19. Tabata Y, Miyao M, Inamoto T, et al. De novo formation of adipose tissue by controlled release of basic fibroblast growth factor. Tissue Eng 6, 279, 2000.

20. Ceradini DJ, Kulkarni AR, Callaghan MJ, et al. Progenitor cell trafficking is regulated by hypoxic gradients through HIF1 induction of SDF-1. Nat Med 10, 858, 2004. 
21. Zuk PA, Zhu M, Mizuno H, et al. Multilineage cells from human adipose tissue: implications for cell-based therapies. Tissue Eng 7, 211, 2001.

22. Varzaneh FE, Shillabeer G, Wong KL, Lau DC. Extracellular matrix components secreted by microvascular endothelial cells stimulate preadipocyte differentiation in vitro. Metabolism 43, 906, 2004.

23. Stefansson S. Lawrence D.A. The serpin PAI-1 inhibits cell migration by blocking integrin alpha $\mathrm{V}$ beta 3 binding to vitronectin. Nature 383, 441, 1996.

24. Pettersson P, Cigolini M, Sjostrom L, Smith U, Bjorntorp P. Cells in human adipose tissue developing into adipocytes. Acta Med Scand 215, 447, 1984.
25. Fukumura D, Ushiyama A, Duda DG, et al. Paracrine regulation of angiogenesis and adipocyte differentiation during in vivo adipogenesis. Circ Res 93, e88, 2003.

Address reprint requests to: Keren Abberton, M. Rep. Sci., Ph.D. Bernard O'Brien Institute of Microsurgery

42 Fitzroy St Fitzroy, Victoria 3065 Australia

E-mail: Abberton@unimelb.edu.au 